ADIPOSITAS

\section{Dick vom Kirchgang?}

- Der Mensch lebt nicht vom Brot allein aber ganz ohne das leibliche Wohl geht es eben auch nicht. Das scheint für fleißige Kirchgänger ganz besonders zuzutreffen, wie die CARDIA-Studie gezeigt hat: Danach sind Menschen, die als junge Erwachsene regelmäßig einen Gottesdienst besucht haben, siginifikant häufiger in ihren mittleren Lebensjahren übergewichtig.

In die Studie waren 2433 Männer und Frauen zwischen 20 und 32 Jahren aufgenommen und nach ihren religiösen $\mathrm{Ge}$ wohnheiten befragt worden. Nach im Schnitt 18 Jahren wurde das kardiovaskuläre Risikoprofil der Probanden erfasst. Übergewicht und Adipositas waren bei den regelmäßigen Kirchgängern besonders häufig. Wer wenigstens einmal pro Woche zum Gottesdienst ging, hatte gegenüber den Nicht-Kirchgängern ein um 50\% erhöhtes Risiko, übergewichtig zu werden. Die Autoren wiesen allerdings darauf hin, dass bei Kirchgängern dafür andere Risikofaktoren wie Rauchen seltener sind und dass sie abgesehen von ihren Gewichtsproblemen in der Regel einen gesünderen Lebensstil aufweisen.

$\mathrm{MO}$ -

Feinstein MJ et al, Cardiovascular Disease Epidemiology and Prevention and Nutrition, Physical Activity and Metabolism 2011 Conference, Atlanta, 23.3.2011, Poster MP016

\title{
LIEBESKUMMER
}

\section{Körperliche Schmerzen durch verletzte Gefühle}

- Das ungewollte Ende einer Liebesbeziehung ist schmerzhaft - im wörtlichen Sinn: Bei der verlassenen Person werden Gehirnregionen aktiviert, die auch an der Empfindung physischer Schmerzen beteiligt sind.

Um den Herzschmerz wissenschaftlich zu fassen zu bekommen, wurden in Manhattan mithilfe von Flugblättern und Anzeigen 40 "frisch" Verlassene requiriert. Die Probanden sollten ein Foto von der/ dem Ex bzw. einer befreundeten Person

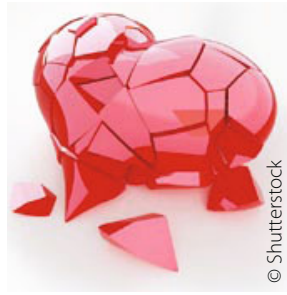

betrachten und jeweils an gemeinsame Erlebnisse denken. Was sich dabei in ihren Gehirnen abspielte, wurde mit dem funktionellem MRT (fMRT) erfasst. Zusätzlich wurde die Auswirkung eines schmerzhaften Hitzereizes per fMRT analysiert. Dabei zeigte sich, dass einige Gehirnregionen, die bei einem physischen Schmerz aktiv sind, auch durch Liebeskummer stimuliert werden. BS = Proc Nat Acad Sci, online 28. März 2011

\section{LEBENSQUALITÄT GEWINNEN}

\section{Antidepressive Therapie nach Schlaganfall verbessert Körperfunktion}

- Etwa jeder dritte Schlaganfallpatient entwickelt eine Depression. Wird diese innerhalb von drei Monaten erfolgreich behandelt, gelingt es den Betroffenen leichter, ihre funktionellen Fähigkeiten wiederzuerlangen. Patienten, bei denen die depressiven Symptome fortbestehen, haben deutlich größere Schwierigkeiten, Alltagstätigkeiten wie Anziehen, Essen etc. selbstständig zu verrichten, ergab eine US-Studie.
Der Zusammenhang zwischen Depression und Verschlechterung der Körperfunktionen nach Schlaganfall ist bereits aus früheren Studien bekannt. Diese Studie ist aber eine der ersten, die zusätzlich zeigt, dass die erfolgreiche Behandlung depressiver Symptome die funktionellen Ergebnisse nach Insult tatsächlich verbessern kann. EO Schmid AA, et al. Neurology 2011;76:1000-1005

\section{SCHLAFLOS NACH DER ARBEIT AM COMPUTER? Wachmacher LED-Bildschirm}

- Wer seinen Abend vor einem mit LEDs ausgerüsteten Fernseher oder PC-Bildschirm verbringt, fühlt sich weniger müde und verzögert seinen Schlaf-Wach-Rhythmus. Dieses Studienergebnis berichten Forscher der Universität Basel und des Fraunhofer-Instituts für Arbeitswirtschaft und Organisation in Stuttgart.

Viele Menschen verbringen gerade abends ihre Zeit vor Bildschirmen (TV, PC oder Laptop), die mit einer LED-Hintergrundbeleuchtung (sog. Backlight) ausgestattet sind. Mit dieser Technik ausgestattete Bildschirme emittieren mehr als doppelt so viel Licht der Wellenlängen zwischen 454 und $474 \mathrm{~nm}$. Licht in diesem Wellenlängenbereich beeinflusst den Spiegel des Schlafhormons Melatonin und die kognitive Leistungsfähigkeit am Abend besonders stark, so Prof. Christian Cajochen, Basel.

Für die Studie verbrachten männliche Versuchspersonen abends fünf Stunden vor LED-Bildschirmen oder vor Monitoren ohne diese Technik. Die Testpersonen vor den LED-Bildschirmen zeigten eine um bis zu $20 \%$ schnellere Reaktionszeit. Sie waren subjektiv und objektiv wacher und wiesen in einem Wortpaarlerntest die besseren Re-

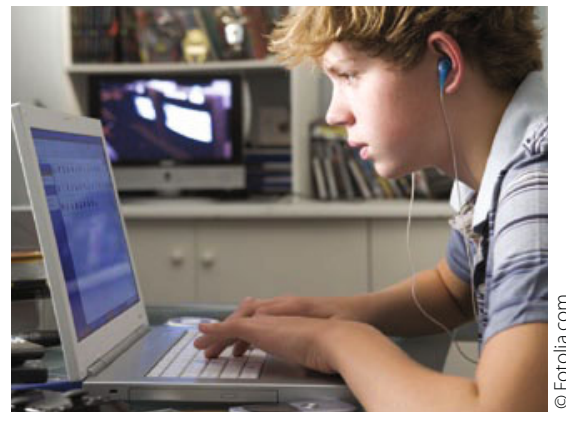

Das Licht von LED-Bildschirmen beeinflusst den Melatoninspiegel.

sultate auf. Zudem war bei innen das Schlafhormon Melatonin länger unterdrückt, d. h. sie konnten erst mit Verspätung einschlafen.

JM .

Journal of Applied Physiology, 2011; doi:10.1152/japplphysiol.00165.2011 